\title{
ATLAS diboson excess from Stueckelberg mechanism
}

\author{
Wan-Zhe Feng, ${ }^{a}$ Zuowei Liu ${ }^{b, c}$ and Pran Nath ${ }^{d}$ \\ ${ }^{a}$ Max-Planck-Institut für Physik (Werner-Heisenberg-Institut), \\ 80805 München, Germany \\ ${ }^{b}$ Department of Physics, Nanjing University, \\ Nanjing, 210093, China \\ ${ }^{c}$ Institute of Modern Physics and Center for High Energy Physics, \\ Tsinghua University, Beijing, 100084, China \\ ${ }^{d}$ Department of Physics, Northeastern University, \\ Boston, MA 02115-5000, U.S.A. \\ E-mail: vicf@mpp.mpg.de, zuoweiliu@nju.edu.cn, nath@neu.edu
}

ABSTRACT: We discuss the diboson excess seen by the ATLAS collaboration around $2 \mathrm{TeV}$ in the LHC run I at $\sqrt{s}=8 \mathrm{TeV}$. We explore the possibility that such an excess can arise from a $Z^{\prime}$ boson which acquires mass through a $\mathrm{U}(1)_{X}$ Stueckelberg extension. The corresponding $Z^{\prime}$ gauge boson is leptophobic with a mass of around $2 \mathrm{TeV}$ and has interactions with $\mathrm{SU}(2)_{L}$ Yang-Mills fields and gauge fields of the hypercharge. The analysis predicts $Z^{\prime}$ decays into $W W$ and $Z Z$ as well as into $Z \gamma$. Further three-body as well as four-body decays of the $Z^{\prime}$ such as $W W Z, W W \gamma, W W Z Z$ etc are predicted. In the analysis we use the helicity formalism which allows us to exhibit the helicity structure of the $Z^{\prime}$ decay processes in an transparent manner. In particular, we are able to show the set of vanishing helicity amplitudes in the decay of the massive $Z^{\prime}$ into two vector bosons due to angular momentum conservation with a special choice of the reference momenta. The residual set of non-vanishing helicity amplitudes are identified. The parameter space of the model compatible with the diboson excess seen by the ATLAS experiment at $\sqrt{s}=8 \mathrm{TeV}$ is exhibited. Estimate of the diboson excess expected at $\sqrt{s}=13 \mathrm{TeV}$ with $20 \mathrm{fb}^{-1}$ of integrated luminosity at LHC run II is also given. It is shown that the $W W, Z Z$ and $Z \gamma$ modes are predicted to be in the approximate ratio $1: \cos ^{2} \theta_{W}\left(1+\alpha \tan ^{2} \theta_{W}\right)^{2} / 2:(1-\alpha)^{2} \sin ^{2} \theta_{W} / 2$ where $\alpha$ is the strength of the coupling of $Z^{\prime}$ with the hypercharge gauge field relative to the coupling with the Yang-Mills gauge fields. Thus observation of the $Z \gamma$ mode as well as three-body and four-body decay modes of the $Z^{\prime}$ will provide a definite test of the model and of a possible new source of interaction beyond the standard model.

KeYwords: Beyond Standard Model, Gauge Symmetry

ARXiv EPrint: 1511.08921 


\section{Contents}

1 Introduction 1

$2 Z^{\prime}$ to Diboson decays 1

$\begin{array}{llr}3 & \text { Phenomenology } & 7\end{array}$

4 Conclusion $\quad 8$

\section{Introduction}

The ATLAS collaboration at CERN [1] has seen a diboson excess around $2 \mathrm{TeV}$ in the $W Z$, $W W$, and $Z Z$ channels with local significance of $3.4 \sigma, 2.6 \sigma$, and $2.9 \sigma$ in that order. In this work we discuss a model where the source of the diboson excess is a $Z^{\prime}$ boson which gains mass through the Stueckelberg mechanism [2-12] and has interactions with $\mathrm{SU}(2)_{L}$ Yang-Mills gauge bosons. Our model differs in significant ways from a variety of other models that have been proposed to explain the excess. These include models with strong dynamics [13], $W^{\prime}$ models [14], models based on strings [15], composite spin zero boson models [16], and many others [17-63].

\section{$2 \quad Z^{\prime}$ to Diboson decays}

We consider a $\mathrm{U}(1)_{X}$ extension of the standard model with $C_{\mu}(x)$ as the gauge boson and we propose the following effective interaction

$$
\mathcal{L}=\frac{1}{\Lambda^{2}}\left[\left(\frac{1}{M} \partial_{\mu} \sigma+C_{\mu}\right) \partial_{\nu}\left(F_{a}^{\mu \lambda} F_{a \lambda}{ }_{\lambda}+\alpha B^{\mu \lambda} B_{\lambda}^{\nu}\right)+(\mu \leftrightarrow \nu)\right],
$$

where $F$ and $B$ are the $\mathrm{SU}(2)_{L}$ and $\mathrm{U}(1)_{Y}$ field strengths, $a$ is the $\mathrm{SU}(2)_{L}$ index, $\alpha$ is the strength of the coupling of $C_{\mu}$ with the hypercharge gauge field relative to the coupling with the Yang-Mills gauge fields and is a free parameter, and the new physics scale $\Lambda$ will be determined by experiment. In eq. (2.1) we are using the Stueckelberg mechanism to make the Lagrangian gauge invariant. This occurs due to the following gauge transformations of $C_{\mu}$ and $\sigma: C_{\mu} \rightarrow C_{\mu}-\partial_{\mu} \lambda, \sigma \rightarrow M \lambda$. Thus the interactions is $\mathrm{U}(1)_{X}$ and $\mathrm{SU}(2)_{L}$ gauge invariant. For more details of Stueckelberg mechanism and the Stueckelberg U(1) extension of standard model or minimal supersymmetric standard model, see [2-4].

In the unitary gauge we define $Z_{\mu}^{\prime}=\left(\frac{1}{M} \partial_{\mu} \sigma+C_{\mu}\right)$. These interactions will describe the possible decays of the $Z^{\prime}$ into two-body, three-body and four-body states. After expansion, the three-point interactions read

$$
\mathcal{L}_{3 \mathrm{pt}}=\frac{2}{\Lambda^{2}} Z_{\mu}^{\prime}\left(\partial^{\nu} A_{a}^{\mu} \partial^{2} A_{a \nu}-\partial^{\mu} A_{a}^{\nu} \partial^{2} A_{a \nu}+\alpha \partial^{\nu} B^{\mu} \partial^{2} B_{\nu}-\alpha \partial^{\mu} B^{\nu} \partial^{2} B_{\nu}\right) .
$$


Further after spontaneous breaking of the electroweak symmetry we will have a Lagrangian describing the interactions of the $Z^{\prime}$ with $W, Z$ and $\gamma$. For the two-body decays the possible modes are $W^{+} W^{-}, Z Z, Z \gamma$. In addition, eq. (2.1) also provides three- and four-body decays such as $W^{+} W^{-} Z, W^{+} W^{-} \gamma$ and $W^{+} W^{-} Z Z$ etc. For the two-body decay, eq. (2.2) further reduces to

$$
\begin{aligned}
\mathcal{L}_{3 \mathrm{pt}}= & \frac{2 m_{W}^{2}}{\Lambda^{2}} Z_{\mu}^{\prime}\left(\partial^{\nu} W^{-\mu} W_{\nu}^{+}+\partial^{\nu} W^{+\mu} W_{\nu}^{-}\right) \\
& +\frac{2 m_{Z}^{2}}{\Lambda^{2}} Z_{\mu}^{\prime}\left[\cos ^{2} \theta_{W} \partial^{\nu} Z^{\mu} Z_{\nu}+\sin \theta_{W} \cos \theta_{W}\left(\partial^{\nu} A^{\mu} Z_{\nu}-\partial^{\mu} A^{\nu} Z_{\nu}\right)\right] \\
& +\frac{2 m_{Z}^{2}}{\Lambda^{2}} \alpha Z_{\mu}^{\prime}\left[\sin ^{2} \theta_{W} \partial^{\nu} Z^{\mu} Z_{\nu}-\sin \theta_{W} \cos \theta_{W}\left(\partial^{\nu} A^{\mu} Z_{\nu}-\partial^{\mu} A^{\nu} Z_{\nu}\right)\right],
\end{aligned}
$$

where $\sin \theta_{W}=g / \sqrt{g^{2}+g^{\prime 2}}, \cos \theta_{W}=g^{\prime} / \sqrt{g^{2}+g^{\prime 2}}$. First we notice $Z^{\prime}$ to diphoton channel automatically vanishes consistent with the Landau-Yang theorem [67-71]. As seen from eq. (2.3) the non-vanishing two-body decays consist of the final states $W^{+} W^{-}, Z Z$, $Z \gamma$. For the case $\alpha=1$ the $Z^{\prime} \rightarrow Z \gamma$ mode vanishes. In the analysis of these final states we will use the helicity formalism. In this formalism the three-point amplitudes for these processes read

$$
\begin{aligned}
\mathscr{A}\left[Z^{\prime}\left(\xi^{\prime}, k^{\prime}\right)\right. & \left.\rightarrow W^{+}\left(\xi_{1}^{+}, k_{1}\right) W^{-}\left(\xi_{2}^{-}, k_{2}\right)\right] \\
& =\frac{2 m_{W}^{2}}{\Lambda^{2}}\left[\left(\xi^{\prime *} \cdot \xi_{2}^{-}\right)\left(\xi_{1}^{+} \cdot k_{2}\right)+\left(\xi^{*} \cdot \xi_{1}^{+}\right)\left(\xi_{2}^{-} \cdot k_{1}\right)\right], \\
\mathscr{A}\left[Z^{\prime}\left(\xi^{\prime}, k^{\prime}\right)\right. & \left.\rightarrow Z\left(\xi_{1}, k_{1}\right) Z\left(\xi_{2}, k_{2}\right)\right] \\
& =\frac{2 m_{Z}^{2}}{\Lambda^{2}}\left(\cos ^{2} \theta_{W}+\alpha \sin ^{2} \theta_{W}\right)\left[\left(\xi^{*} \cdot \xi_{2}\right)\left(\xi_{1} \cdot k_{2}\right)+\left(\xi^{\prime *} \cdot \xi_{1}\right)\left(\xi_{2} \cdot k_{1}\right)\right], \\
\mathscr{A}\left[Z^{\prime}\left(\xi^{\prime}, k^{\prime}\right)\right. & \left.\rightarrow Z(\xi, k) \gamma\left(\epsilon, k_{0}\right)\right] \\
& =\frac{2 m_{Z}^{2}}{\Lambda^{2}}(1-\alpha) \sin \theta_{W} \cos \theta_{W}\left[\left(\xi^{\prime *} \cdot \epsilon\right)\left(\xi \cdot k_{0}\right)-\left(\xi^{* *} \cdot k_{0}\right)(\xi \cdot \epsilon)\right],
\end{aligned}
$$

where $\xi^{\prime}, \xi_{ \pm}, \xi, \epsilon$ denote the polarization vector of $Z^{\prime}, W^{ \pm}, Z, \gamma$. To see the helicity structure of the above amplitudes more explicitly, we now apply the spinor helicity formalism. A massless spin one gauge boson has two degrees of freedom, corresponding to up and down helicities. They are expressed by the polarization vectors $\epsilon_{\mu}^{+}$and $\epsilon_{\mu}^{-}$and can be written as [64]

$$
\epsilon_{\mu}^{+}(k, r)=\frac{r_{\dot{a}}^{*} \bar{\sigma}_{\mu}^{\dot{a} a} k_{a}}{\sqrt{2} r_{\dot{a}}^{*} k^{* \dot{a}}}, \quad \epsilon_{\mu}^{-}(k, r)=\frac{k_{\dot{a}}^{*} \bar{\sigma}_{\mu}^{\dot{a} a} r_{a}}{\sqrt{2} k^{a} r_{a}}
$$

where $k$ is the momentum of the particle and $r$ is the reference momentum which can be chosen to be any light-like momentum except $k$. Here the momenta with spinor indices are 2-component commutative spinors, and they are defined as $p_{\mu} \sigma_{a \dot{a}}^{\mu}=-p_{a} p_{\dot{a}}^{*}$. It's easy to show $\epsilon_{+}^{\mu}(k, r)-\epsilon_{+}^{\mu}(k, \tilde{r}) \sim k^{\mu}$ where $\tilde{r}$ is some other free chosen reference momentum. Since the whole amplitude is invariant under the gauge transformation $\epsilon^{\mu} \rightarrow \epsilon^{\mu}+\lambda k^{\mu}$, choosing different reference momentum for a massless gauge boson does not change the result.

A massive spin one gauge boson which is expressed by its polarization vector $\xi_{\mu}$, contains three degrees of freedom associated to the eigenstates of $J_{z}$, where the transversality 
condition $\xi_{\mu} k^{\mu}=0$ eliminates one degree of freedom of the four-vector. The choice of the quantization axis $\vec{z}$ can be handled in an elegant way by decomposing the momentum $k^{\mu}$ into two arbitrary light-like reference momenta $p$ and $q$ :

$$
k^{\mu}=p^{\mu}+q^{\mu}, \quad k^{2}=-m^{2}=2 p q, \quad p^{2}=q^{2}=0 .
$$

Once the reference momenta $p$ and $q$ are chosen, the spin quantization axis of the polarization vector $\xi_{\mu}$ is set to be collinear to the direction of $\vec{q}$ in the rest frame. The 3 spin wave functions depend on $p$ and $q$, while this dependence would drop out in the squared amplitudes summing over all spin directions. The massive spin one wave functions $\xi_{\mu}$ are given by the following polarization vectors (up to a phase factor) $[65,66]$

$$
\begin{aligned}
& \xi^{\mu}\left(k, J_{z}=+1\right)=\frac{1}{\sqrt{2} m} p_{\dot{a}}^{*} \bar{\sigma}^{\mu \dot{a} a} q_{a}, \\
& \xi^{\mu}\left(k, J_{z}=0\right)=\frac{1}{2 m} \bar{\sigma}^{\mu \dot{a} a}\left(p_{\dot{a}}^{*} p_{a}-q_{\dot{a}}^{*} q_{a}\right), \\
& \xi^{\mu}\left(k, J_{z}=-1\right)=-\frac{1}{\sqrt{2} m} q_{\dot{a}}^{*} \bar{\sigma}^{\mu \dot{a} a} p_{a} .
\end{aligned}
$$

With different choices of reference momenta $(p, q)$, one will get different helicity amplitudes. While the $(p, q)$-dependence would drop off when one adds up all the squared helicity amplitudes. Since we have the freedom to choose reference momenta for the interacting spin one gauge bosons, with a clever choice one can not only simplify the computation dramatically but also exhibit the helicity structure in a transparent manner.

For the process $Z^{\prime}\left(k^{\prime}\right) \rightarrow Z(k) \gamma\left(k_{0}\right)$, since the spin quantization axis of a massless photon is collinear to its moving direction, we choose the following reference momenta

$$
\begin{array}{rrl}
\gamma & \epsilon\left(k_{0}\right): & r, \\
Z & \xi(k): & k=r+a k_{0}, \\
Z^{\prime} & \xi^{\prime}\left(k^{\prime}\right): & k^{\prime}=r+(a+1) k_{0},
\end{array}
$$

where $r^{2}=0$ and $a=m_{Z}^{2} /\left(M_{Z^{\prime}}^{2}-m_{Z}^{2}\right)$. For this clever choice, the spin quantization axes of both $Z^{\prime}$ and $Z$ are aligned to the photon moving direction, i.e., the direction of $\vec{k}_{0}$.

For $Z^{\prime}$ decay into two massive gauge bosons with a common mass $m_{i}$, where $m_{i}=m_{W}$ for eq. (2.4) and $m_{i}=m_{Z}$ for eq. (2.5), we choose the following reference momenta

$$
\begin{array}{ccl}
Z_{1} / W^{+} & \xi_{1}\left(k_{1}\right): & k_{1}=c p+q, \\
Z_{2} / W^{-} & \xi_{2}\left(k_{2}\right): & k_{2}=p+c q, \\
Z^{\prime} & \xi^{\prime}\left(k^{\prime}\right): & k^{\prime}=(1+c) p+(1+c) q,
\end{array}
$$

with $m_{i}^{2}=k_{1}^{2}=k_{2}^{2}=2 c p \cdot q, M_{Z^{\prime}}^{2}=k^{\prime 2}=2(1+c)^{2} p \cdot q$, and thus

$$
c_{i}=\frac{1}{2}\left(b_{i}-2\right) \pm \frac{1}{2} \sqrt{b_{i}^{2}-4 b_{i}},
$$

where $b_{i}=M_{Z^{\prime}}^{2} / m_{i}^{2}$. Under this choice of reference momenta, the spin quantization axes of all these three massive gauge bosons are aligned to the same direction, i.e., the direction of $\vec{q}$. 
In sum, for the two cases discussed above the spin quantization axes of the decaying massive gauge boson $Z^{\prime}$ as well as the two gauge bosons in the final state, are aligned to the same direction. Thus for the process $Z^{\prime} \rightarrow Z \gamma$, it is not difficult to show the vanishing of the following helicity amplitudes

$$
\begin{gathered}
\mathscr{A}\left[Z^{\prime} ; Z(+), \gamma(+)\right]=\mathscr{A}\left[Z^{\prime} ; Z(-), \gamma(-)\right]=0, \\
\mathscr{A}\left[Z^{\prime}( \pm) ; Z(+), \gamma(-)\right]=\mathscr{A}\left[Z^{\prime}( \pm) ; Z(-), \gamma(+)\right]=0,
\end{gathered}
$$

as a result of angular momentum conservation, cf., the left panel of figure 1. While the non-vanishing helicity amplitudes are

$$
\begin{aligned}
\mathscr{A}_{1} & \equiv \mathscr{A}\left[Z^{\prime}(+) ; Z(0), \gamma(+)\right]=\mathscr{A}\left[Z^{\prime}(-) ; Z(0), \gamma(-)\right] \\
& =\frac{\sin \theta_{W} \cos \theta_{W}}{\Lambda^{2}} \beta m_{Z}\left(M_{Z^{\prime}}^{2}-m_{Z}^{2}\right), \\
\mathscr{A}_{2} & \equiv \mathscr{A}\left[Z^{\prime}(0) ; Z(+), \gamma(-)\right]=\mathscr{A}\left[Z^{\prime}(0) ; Z(-), \gamma(+)\right] \\
& =\frac{\sin \theta_{W} \cos \theta_{W}}{\Lambda^{2} M_{Z^{\prime}}^{2}} \beta m_{Z}^{2}\left(M_{Z^{\prime}}^{2}-m_{Z}^{2}\right),
\end{aligned}
$$

where $\beta \equiv 1-\alpha$. Thus the total squared-amplitude read

$$
\begin{aligned}
Z^{\prime} \rightarrow Z \gamma: \quad \sum|\mathscr{A}|^{2} & =2\left|\mathscr{A}_{1}\right|^{2}+2\left|\mathscr{A}_{2}\right|^{2} \\
& =\frac{2 \sin ^{2} \theta_{W} \cos ^{2} \theta_{W} \beta^{2}}{\Lambda^{4}} \frac{m_{Z}^{2}}{M_{Z^{\prime}}^{2}}\left(M_{Z^{\prime}}^{2}+m_{Z}^{2}\right)\left(M_{Z^{\prime}}^{2}-m_{Z}^{2}\right)^{2}
\end{aligned}
$$

The factor 2 in $2\left|\mathscr{A}_{1}\right|^{2}$ and $2\left|\mathscr{A}_{2}\right|^{2}$ arise because for each there are two helicity configurations, cf., eqs. (2.21), (2.22), that are non-vanishing.

Next we discuss the $Z^{\prime}$ decay into two massive gauge bosons. An analysis similar to the above gives

$$
\begin{aligned}
\mathscr{A}\left[Z^{\prime}(+) ;+,+\right] & =\mathscr{A}\left[Z^{\prime}(+) ;-,-\right]=\mathscr{A}\left[Z^{\prime}(+) ; 0,0\right]=\mathscr{A}\left[Z^{\prime}(+) ;+,-\right]=0, \\
\mathscr{A}\left[Z^{\prime}(-) ;-,-\right] & =\mathscr{A}\left[Z^{\prime}(-) ;+,+\right]=\mathscr{A}\left[Z^{\prime}(-) ; 0,0\right]=\mathscr{A}\left[Z^{\prime}(-) ;+,-\right]=0, \\
\mathscr{A}\left[Z^{\prime}(0) ;+,+\right] & =\mathscr{A}\left[Z^{\prime}(0) ;-,-\right]=\mathscr{A}\left[Z^{\prime}(0) ;+, 0\right]=\mathscr{A}\left[Z^{\prime}(0) ;-, 0\right]=0, \\
\mathscr{A}\left[Z^{\prime}(0) ;+,-\right] & =\mathscr{A}\left[Z^{\prime}(0) ;-,+\right]=\mathscr{A}\left[Z^{\prime}(0) ; 0,0\right]=0,
\end{aligned}
$$

where the first three lines are due to angular momentum conservation, cf., the left panel of figure 2 and the last line is due to the special choice of the reference momenta eqs. (2.15) to (2.17). The residual set of non-vanishing helicity amplitudes are

$$
\mathscr{A}_{3} \equiv \mathscr{A}\left[Z^{\prime}(+) ;+, 0\right]=\mathscr{A}\left[Z^{\prime}(+) ; 0,+\right]=\mathscr{A}\left[Z^{\prime}(-) ;-, 0\right]=\mathscr{A}\left[Z^{\prime}(-) ; 0,-\right] .
$$

Explicitly they are given by

$$
\begin{aligned}
& \mathscr{A}_{3 W} \equiv \mathscr{A}\left(Z^{\prime} \rightarrow W^{+} W^{-}\right)=\frac{m_{W}^{4}}{\Lambda^{2} M_{Z^{\prime}}} \frac{\left(c_{W}+1\right)^{2}\left(c_{W}-1\right)}{c_{W}^{3 / 2}}, \\
& \mathscr{A}_{3 Z} \equiv \mathscr{A}\left(Z^{\prime} \rightarrow Z Z\right)=\frac{\left(\cos ^{2} \theta_{W}+\alpha \sin ^{2} \theta_{W}\right) m_{Z}^{4}}{\Lambda^{2} M_{Z^{\prime}}} \frac{\left(c_{Z}+1\right)^{2}\left(c_{Z}-1\right)}{c_{Z}^{3 / 2}},
\end{aligned}
$$



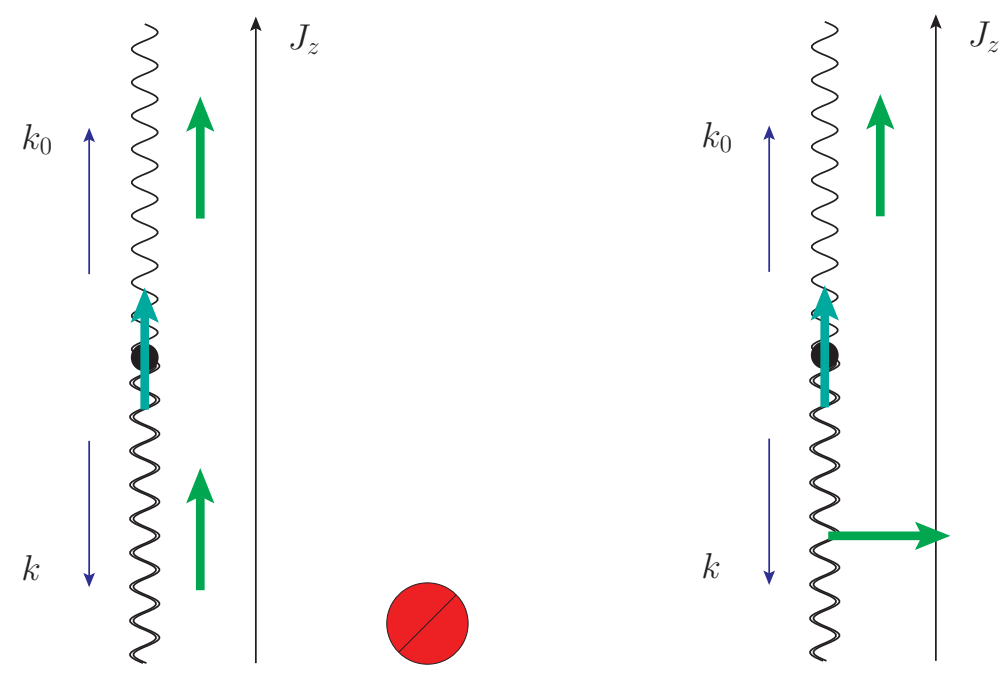

Figure 1. The black dots in the center denote the decaying $Z^{\prime}$. In the center of mass frame, after decay the photon and $Z$ in the final state are moving to the opposite directions. The single wavy lines present the photon and the double wavy lines present the $Z$. The spin of the photon is along (or opposite to) its moving direction. We choose the reference momenta of the massive $Z$ as well as the $Z^{\prime}$ in such a way that their spin quantization axes $\left(\vec{J}_{z}\right)$ are aligned to the photon moving direction. The emerald arrows present the spin of the $Z^{\prime}$, and the green arrows show the spin of the photon and $Z$. The left panel presents the vanishing helicity amplitude $\mathscr{A}\left[Z^{\prime}(+) ; Z(+), \gamma(+)\right]=0$ and this process is not allowed as indicated by the red forbidden sign. The right panel shows the non-vanishing helicity amplitude $\mathscr{A}\left[Z^{\prime}(+) ; Z(0), \gamma(+)\right]$ and this process is allowed as indicated by the green check sign.

where the coefficients $c_{i}$ are given in eq. (2.18), and $m_{i}=m_{W}$ for eq. (2.29) and $m_{i}=m_{Z}$ for eq. (2.30). The total squared-amplitudes read

$$
\begin{aligned}
Z^{\prime} \rightarrow W^{+} W^{-}: \quad \sum|\mathscr{A}|^{2} & =4\left|\mathscr{A}_{3 W}\right|^{2}=\frac{4}{\Lambda^{4}} M_{Z^{\prime}}^{2} m_{W}^{2}\left(M_{Z^{\prime}}^{2}-4 m_{W}^{2}\right), \\
Z^{\prime} \rightarrow Z Z & : \quad \sum|\mathscr{A}|^{2}=2\left|\mathscr{A}_{3 Z}\right|^{2}=\frac{2\left(\cos ^{2} \theta_{W}+\alpha \sin ^{2} \theta_{W}\right)^{2}}{\Lambda^{4}} M_{Z^{\prime}}^{2} m_{Z}^{2}\left(M_{Z^{\prime}}^{2}-4 m_{Z}^{2}\right) .
\end{aligned}
$$

Here the factor 4 for $W^{+} W^{-}$channel is due to the fact that there are in total 4 nonvanishing helicity amplitudes in this channel, cf., eq. (2.28). The factor 2 for $Z Z$ channel is due to the fact that there are only 2 non-vanishing helicity amplitudes since the two final state particles are identical, e.g., $\mathscr{A}\left[Z^{\prime}(+) ;+, 0\right]$ and $\mathscr{A}\left[Z^{\prime}(+) ; 0,+\right]$ give the same amplitude for $Z^{\prime} \rightarrow Z Z$ channel.

In summary, by using the helicity formalism, we can see clearly which helicity modes are forbidden as a result of angular momentum conservation, cf., eqs. (2.19), (2.20) and (2.24) to (2.26) and also figures 1 and 2 . In addition, there are multiple helicity amplitudes which vanish due to the clever choice of the reference momenta, cf., eq. (2.27). 

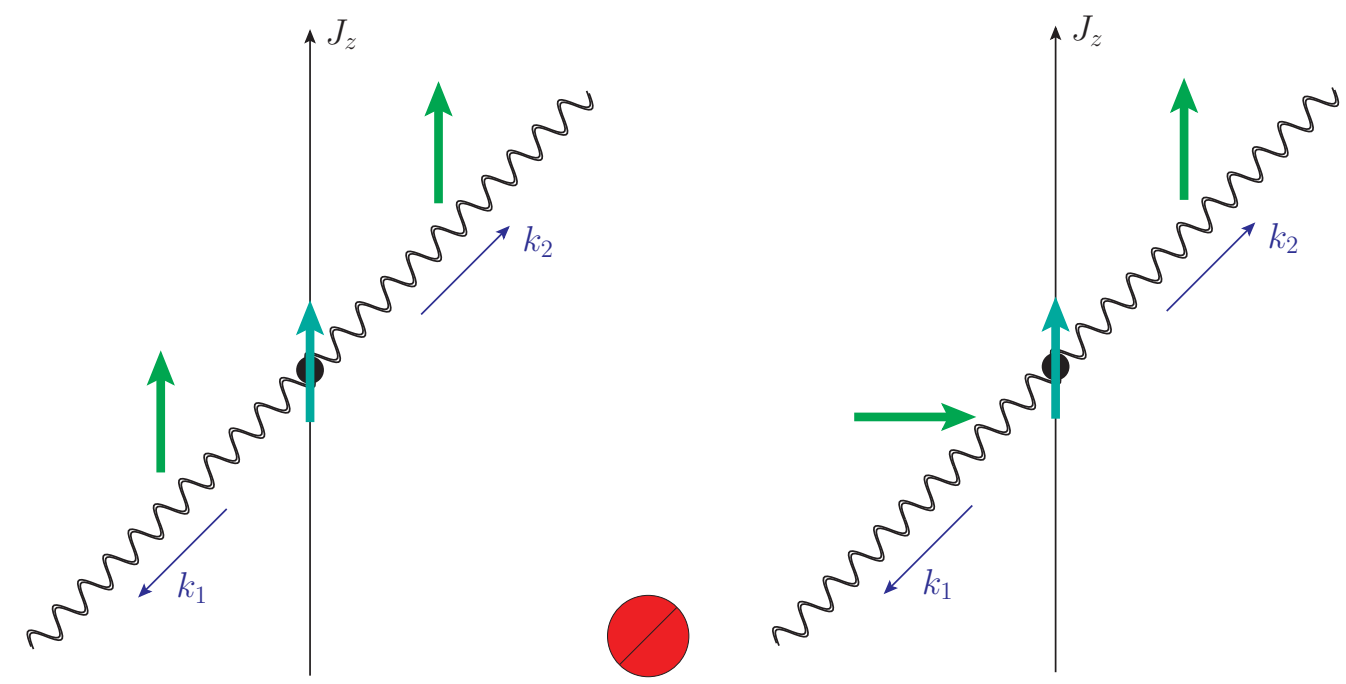

Figure 2. In the center of mass frame, after the decay the two massive gauge bosons in the final state are moving to the opposite directions. The black dots in the center again denote the decaying $Z^{\prime}$. We have chosen the reference momenta in such a way that the spin quantization axes of these three gauge bosons are aligned to the same direction, i.e., $\vec{J}_{z}$. In general, for a massive gauge boson, the direction of $\vec{J}_{z}$ is different with its moving direction because one can always boost it to other reference frames. The emerald arrows present the spin of the $Z^{\prime}$, and the green arrows show the spin of the two final state gauge bosons. The left panel presents the vanishing helicity amplitude $\mathscr{A}\left[Z^{\prime}(+) ;+,+\right]$, and the right panel shows the non-vanishing helicity amplitude $\mathscr{A}\left[Z^{\prime}(+) ; 0,+\right]$.

Using the above the partial decay widths for the processes $W W, Z Z, Z \gamma$ are given by

$$
\begin{aligned}
\Gamma\left(Z^{\prime} \rightarrow W^{+} W^{-}\right) & =\frac{1}{4 \pi} \frac{m_{W}^{2}\left(M_{Z^{\prime}}^{2}-4 m_{W}^{2}\right)^{3 / 2}}{\Lambda^{4}}, \\
\Gamma\left(Z^{\prime} \rightarrow Z Z\right) & =\frac{\left(\cos ^{2} \theta_{W}+\alpha \sin ^{2} \theta_{W}\right)^{2}}{8 \pi} \frac{m_{Z}^{2}\left(M_{Z^{\prime}}^{2}-4 m_{Z}^{2}\right)^{3 / 2}}{\Lambda^{4}}, \\
\Gamma\left(Z^{\prime} \rightarrow Z \gamma\right) & =\frac{(1-\alpha)^{2} \sin ^{2} \theta_{W} \cos ^{2} \theta_{W}}{8 \pi} \frac{m_{Z}^{2}\left(M_{Z^{\prime}}^{2}+m_{Z}^{2}\right)\left(M_{Z^{\prime}}^{2}-m_{Z}^{2}\right)^{3}}{\Lambda^{4} M_{Z^{\prime}}^{5}} .
\end{aligned}
$$

In the limit $M_{Z^{\prime}}^{2} \gg m_{W}^{2}, m_{Z}^{2}$ which holds to better than $1 \%$ accuracy one has the following ratio among the three decay modes

$$
\Gamma\left(W^{+} W^{-}\right): \Gamma(Z Z): \Gamma(Z \gamma) \simeq 2: \cos ^{2} \theta_{W}\left(1+\alpha \tan ^{2} \theta_{W}\right)^{2}:(1-\alpha)^{2} \sin ^{2} \theta_{W} .
$$

For the case $\alpha=0$, the above ratio reduces to

$$
\Gamma\left(W^{+} W^{-}\right): \Gamma(Z Z): \Gamma(Z \gamma) \simeq 2: \cos ^{2} \theta_{W}: \sin ^{2} \theta_{W},
$$

and for the case $\alpha=1$, the above ratio gives

$$
\Gamma\left(W^{+} W^{-}\right): \Gamma(Z Z): \Gamma(Z \gamma) \simeq 2: \cos ^{-2} \theta_{W}: 0 .
$$

Thus we see that both the $Z Z$ and the $Z \gamma$ modes are highly model dependent and they could be vanishing or non-vanishing depending on the value of $\alpha$ (which can be either positive or negative); more LHC data are needed to fully discriminate the three diboson channels and to fix the $\alpha$ parameter. 


\section{Phenomenology}

Regarding the coupling of the $Z^{\prime}$ to the standard model fermions, we will assume a leptophobic $Z^{\prime}$ with the following direct interaction to quarks

$$
\mathcal{L}_{\text {int }}=g_{X} Z_{\mu}^{\prime} \bar{q} \gamma^{\mu} q
$$

The decay width to quarks due to the direct couplings is given by

$$
\Gamma_{\text {direct }}=N_{c} N_{f} g_{X}^{2} \frac{M_{Z^{\prime}}}{12 \pi},
$$

where $N_{c}=3$ is the QCD color factor, and $N_{f}$ is the number of quark flavors that the $Z^{\prime}$ can decay into which is the number of kinematically allowed flavors. Without going into details we assume that our $\mathrm{U}(1)_{X}$ with a gauged baryon number is anomaly free. Such a $\mathrm{U}(1)_{X}$ can arise in a variety of settings such as from gut models [72], or anomaly-free family-dependent U(1)'s [73], with extra heavy chiral particles to cancel the anomaly [7477]. We further assume that the heavy chiral states are not accessible at the current LHC energy and thus do not enter in $Z^{\prime}$ decay.

We discuss now the production cross section of the $Z^{\prime}$ at LHC at $\sqrt{s}=8 \mathrm{TeV}$ and estimate the size of the diboson excess. The parton level cross section for the process $q \bar{q} \rightarrow Z^{\prime} \rightarrow W^{+} W^{-}$using Breit-Wigner form for the $Z^{\prime}$ intermediate state is given by

$$
\hat{\sigma}\left(q \bar{q} \rightarrow Z^{\prime} \rightarrow W^{+} W^{-}\right)=\frac{1}{12 \pi \Lambda_{\mathrm{eff}}^{4}} \sqrt{1-4 m_{W}^{2} / \hat{s}} \frac{\hat{s} M_{Z^{\prime}}^{2}\left(\hat{s}-4 M_{Z^{\prime}}^{2} x_{W}^{2}\right) x_{W}^{2}}{\left(\hat{s}-M_{Z^{\prime}}^{2}\right)^{2}+\hat{s}^{2} \Gamma_{Z^{\prime}}^{2} M_{Z^{\prime}}^{-2}},
$$

where $\Lambda_{\text {eff }}^{2}=\Lambda^{2} / g_{X}, x_{W} \equiv m_{W} / m_{Z^{\prime}}$. The $q \bar{q} \rightarrow Z^{\prime} \rightarrow Z Z$ cross section can be obtained easily if one replaces $m_{W}$ by $m_{Z}$ and inserts the overall factor $\left(\cos ^{2} \theta_{W}+\alpha \sin ^{2} \theta_{W}\right)^{2} / 2$ to the above $W^{+} W^{-}$cross section. The hadron cross section at the $\mathrm{LHC}(\sqrt{s}=8 \mathrm{TeV})$ is computed via the convolution

$$
\sigma\left(p p \rightarrow Z^{\prime} \rightarrow W^{+} W^{-}\right)=K \int d \tau \hat{\sigma}(\hat{s}=s \tau) \frac{d \mathcal{L}}{d \tau}(\tau)
$$

where we use $K \simeq 1.3[78,79]$ to approximate the next to leading correction and $d \mathcal{L} / d \tau$ is the parton luminosity given by $d \mathcal{L} / d \tau(\tau)=2 \int_{\tau}^{1}(d x / x)[u(x) \bar{u}(\tau / x)+d(x) \bar{d}(\tau / x)]$.

The most stringent LHC constraints for the leptophobic $Z^{\prime}$ come from the $t \bar{t}$ resonance search $[80,81]$, and the dijet channel $[82,83]$. The 95\% CL upper limit on dijet cross section for a $2 \mathrm{TeV} Z^{\prime}$ is $\sigma\left(Z^{\prime}\right) \times \mathrm{BR}\left(Z^{\prime} \rightarrow q \bar{q}\right)<100 \mathrm{fb}$ [82]. For $t \bar{t}$ resonance search, the $95 \%$ CL upper limit for a $2 \mathrm{TeV} Z^{\prime}$ is $\sigma\left(Z^{\prime}\right) \times \mathrm{BR}\left(Z^{\prime} \rightarrow t \bar{t}\right)<11(18) \mathrm{fb}$ when $\Gamma_{Z^{\prime}}=20$ (200) $\mathrm{GeV}$ [80]. We use $11 \mathrm{fb}$ as the limit in the $t \bar{t}$ channel for $\Gamma_{Z^{\prime}} \leq 20 \mathrm{GeV}, 18 \mathrm{fb}$ for $\Gamma_{Z^{\prime}} \geq$ $200 \mathrm{GeV}$, and linearly interpolate these two values for decay widths in between. Because the $Z^{\prime}$ boson couples universally to all quarks in our model, the current $t \bar{t}$ constraint turns to be almost always stronger than the current dijet constraint at the LHC. Thus, we only consider the $t \bar{t}$ constraint in our analysis.

An analysis of the diboson excess in the $\Lambda_{\text {eff }}^{2}-g_{X}$ plane is exhibited in figure 3 . Thus the left panel of figure 3 gives the prediction of the model at $\sqrt{s}=8 \mathrm{TeV}$. The red solid 

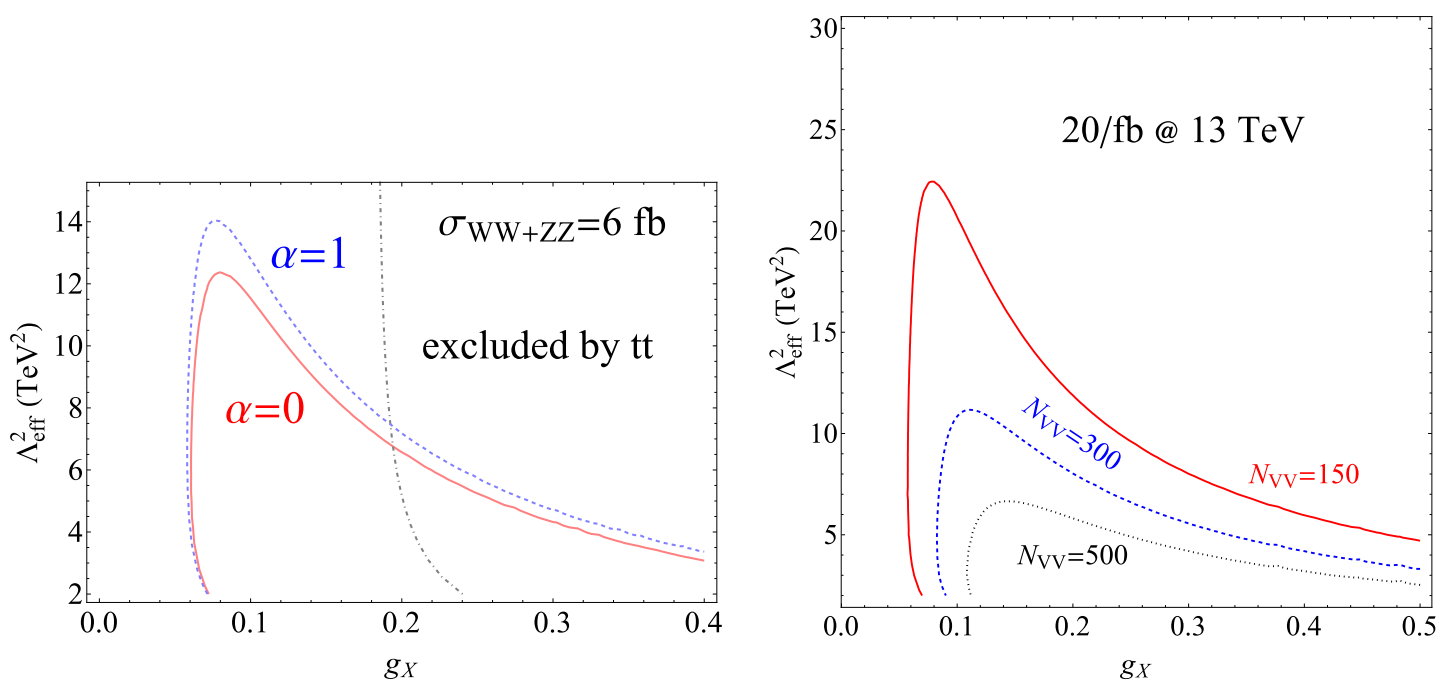

Figure 3. Left panel: analysis at $\sqrt{s}=8 \mathrm{TeV}$ using events over the energy range $1.8 \mathrm{TeV}<\sqrt{\hat{s}}<$ $2.2 \mathrm{TeV}$ computed for both $\alpha=0$ and $\alpha=1$ cases: the red solid (blue dashed) curve predicts $\sigma_{\mathrm{WW}+\mathrm{ZZ}}=6 \mathrm{fb}$ for the $\alpha=0(\alpha=1)$ case, where the Breit-Wigner effects of the $Z^{\prime}$ resonance have been taken into account. The parameter region on the right hand side of the gray dot-dashed curve is excluded by the $t \bar{t}$ resonance search at the LHC. Right panel: expected number of events in the energy range $1.8 \mathrm{TeV}<m_{V V}<2.2 \mathrm{TeV}$ with an integrated luminosity $20 \mathrm{fb}^{-1}$ at LHC run II at $\sqrt{s}=13 \mathrm{TeV}$. Here $V V$ includes both $W W$ and $Z Z$ events. We take $\alpha=0$ here, and assume the same event selection efficiency with event topology requirements as given in the ATLAS analysis [1], which is $\sim 80 \%$.

curve gives rise to a diboson cross section $\sigma_{\mathrm{WW}+\mathrm{ZZ}}=6 \mathrm{fb}$ at $\mathrm{LHC}$ for $\sqrt{s}=8 \mathrm{TeV}$, where $\alpha=0$ is taken.

For comparison we give the analysis by taking $\alpha=1$ which is shown by the blue dashed curve which are shifted upward relative to the red solid curve, in the left panel of figure 3. A prediction of what we will see at $\sqrt{s}=13 \mathrm{TeV}$ at the LHC run II is given in the right panel of figure 3 in terms of the expected number of events at an integrated luminosity of $20 \mathrm{fb}^{-1}$. The LHC production cross section at $13 \mathrm{TeV}$ of the $2 \mathrm{TeV} Z^{\prime}$ boson in our model is about 7 times larger than at $8 \mathrm{TeV}: \sigma_{13 \mathrm{TeV}} / \sigma_{8 \mathrm{TeV}} \simeq 7$. In the LHC run II results recently released, the diboson excess events near $2 \mathrm{TeV}$ observed at the $8 \mathrm{TeV}$ data are not seen in the new $13 \mathrm{TeV}$ data [84, 85]. However, because the new ATLAS (CMS) data consist of an integrated luminosity of $\sim 3.2(2.6) \mathrm{fb}^{-1}$ only, its discovery potential is not improved compared to the $8 \mathrm{TeV}$ data with $20 \mathrm{fb}^{-1}$ integrated luminosity. Thus we take an agnostic attitude towards the diboson excess events and await future LHC data with larger luminosity to sort this anomaly out. If less excess events are seen in the future data, the parameter $\Lambda_{\text {eff }}$ should be increased to larger values for a given $g_{X}$ value.

\section{Conclusion}

In this work we have investigated the diboson excess seen at ATLAS via the decay of a leptophobic Stueckelberg $Z^{\prime}$ boson with a mass around $2 \mathrm{TeV}$. It is possible to accommodate 
the diboson excess seen by the ATLAS collaboration within the model of eq. (2.1). Further, the model makes the prediction of a $Z \gamma$ mode which should also be seen. Additionally the model predicts three-body decays such as $W W Z, W W \gamma$ and four-body decay modes such as $W W W W, W W Z Z, W W Z \gamma$ etc. Observation of such modes would provide a confirmation of the proposed model. We also make estimates of the diboson cross sections at LHC run II.

The proposed model contains new interactions involving vertices $Z^{\prime} Z Z, Z^{\prime} W W, Z^{\prime} Z \gamma$ which can contribute to the oblique parameters. Specifically, corrections to the parameter $S$ can arise from the $Z-Z, \gamma \gamma$ and $Z-\gamma$ self-energy diagrams [86]. From eq. (2.3) we see that the effective coupling is of the size $g_{\text {eff }} \sim \mathcal{O}\left(M_{Z}^{2} / \Lambda^{2}\right)$. Taking $\Lambda^{2} \sim 10 \mathrm{TeV}^{2}$, we have that $g_{\text {eff }} \sim 10^{-3}$ and the loop is proportional to $\alpha_{\text {eff }}=g_{\text {eff }}^{2} /(4 \pi) \sim 10^{-7}$. This is to be compared to the electroweak fine-structure constant $\alpha_{2} \sim 0.033$. Thus the contribution from the new physics loops to the $S$ parameter would be much smaller that the current error corridor on $S$ as can be seen from the global gfitter [87] results which give $S=0.05 \pm 0.11$.

The proposed interaction eq. (2.1) is phenomenological and it should be interesting to look for an ultraviolet complete model that can give rise to such an interaction if the results of LHC run I are confirmed in LHC run II. Our purpose in investigating the model of eq. (2.1) is to show that there exists an interaction which could produce the desired diboson resonance. The largeness of the effect seen demands that the effective scale $\Lambda$ be not too high. A more fundamental model which replaces eq. (2.1) would only readjust the parameters but our main hypothesis that any fundamental interaction that can produce eq. (2.1) can explain the experimental observation would still hold. An interesting attribute of eq. (2.1) is that it is a CP-violating interaction and thus a check of this model and specifically of eqs. (2.36) to (2.38) implies that one is testing a new source of $\mathrm{CP}$ violation which is accessible at LHC energies. We note that $\Lambda$ is not necessarily the mass of a field but a composite scale, and the mass of the heavy field that gives rise to eq. (2.1) could be much higher. Consider, for example, a two-index field $\sigma_{\mu \nu}$ with a Lagrangian interaction $\mathcal{L} \sim m_{1}^{2} \sigma_{\mu \nu} \sigma^{\mu \nu}+m_{2}^{-1} \sigma_{\mu \nu} J^{\mu \nu}$ with $J^{\mu \nu}=F^{\mu \lambda} F_{\lambda}^{\nu}+m_{3}^{2}\left(\partial^{\mu} C^{\nu}+\partial^{\nu} C^{\mu}\right)$. Integration on the $\sigma$ field leads to the interaction of eq. (2.1) with $\Lambda \sim m_{1} m_{2} / m_{3}$. It is clear that the choice $m_{3} / m_{2} \simeq 6$ will lead to $m_{1} \sim 10 \mathrm{TeV}$, i.e., the mass of the heavy field would be significantly higher than the resonance mass.

\section{Acknowledgments}

WZF is grateful to Yang Zhang for helpful discussions. WZF is supported by the Alexander von Humboldt Foundation and Max-Planck-Institut für Physik, München. The work of Z.L. is supported in part by the Tsinghua University Grant 523081007. The work of PN is supported in part by the U.S. National Science Foundation (NSF) grant PHY-1314774.

Open Access. This article is distributed under the terms of the Creative Commons Attribution License (CC-BY 4.0), which permits any use, distribution and reproduction in any medium, provided the original author(s) and source are credited. 


\section{References}

[1] ATLAS collaboration, Search for high-mass diboson resonances with boson-tagged jets in proton-proton collisions at $\sqrt{s}=8$ TeV with the ATLAS detector, JHEP 12 (2015) 055 [arXiv: 1506.00962] [INSPIRE].

[2] B. Körs and P. Nath, A Stueckelberg extension of the standard model, Phys. Lett. B 586 (2004) 366 [hep-ph/0402047] [INSPIRE].

[3] B. Körs and P. Nath, A supersymmetric Stueckelberg U(1) extension of the MSSM, JHEP 12 (2004) 005 [hep-ph/0406167] [INSPIRE].

[4] B. Körs and P. Nath, Aspects of the Stueckelberg extension, JHEP 07 (2005) 069 [hep-ph/0503208] [INSPIRE].

[5] K. Cheung and T.-C. Yuan, Hidden fermion as milli-charged dark matter in Stueckelberg $Z^{\prime}$ model, JHEP 03 (2007) 120 [hep-ph/0701107] [INSPIRE].

[6] D. Feldman, Z. Liu and P. Nath, The Stueckelberg extension and milli weak and milli charged dark matter, AIP Conf. Proc. 939 (2007) 50 [arXiv:0705.2924] [InSPIRE].

[7] D. Feldman, B. Körs and P. Nath, Extra-weakly interacting dark matter, Phys. Rev. D 75 (2007) 023503 [hep-ph/0610133] [INSPIRE].

[8] Z. Liu, P. Nath and G. Peim, An explanation of the CDF dijet anomaly within a $U(1)_{X}$ Stueckelberg extension, Phys. Lett. B 701 (2011) 601 [arXiv:1105.4371] [InSPIRE].

[9] N. Chen, Z. Liu and P. Nath, $3.5 \mathrm{keV}$ galactic emission line as a signal from the hidden sector, Phys. Rev. D 90 (2014) 035009 [arXiv:1406.0687] [INSPIRE].

[10] W.-Z. Feng, P. Nath and G. Peim, Cosmic coincidence and asymmetric dark matter in a Stueckelberg extension, Phys. Rev. D 85 (2012) 115016 [arXiv:1204.5752] [INSPIRE].

[11] W.-Z. Feng, G. Shiu, P. Soler and F. Ye, Probing hidden sectors with Stückelberg U(1) gauge fields, Phys. Rev. Lett. 113 (2014) 061802 [arXiv:1401.5880] [INSPIRE].

[12] W.-Z. Feng, G. Shiu, P. Soler and F. Ye, Building a Stückelberg portal, JHEP 05 (2014) 065 [arXiv: 1401.5890] [INSPIRE].

[13] H.S. Fukano, M. Kurachi, S. Matsuzaki, K. Terashi and K. Yamawaki, 2 TeV walking Technirho at LHC?, Phys. Lett. B 750 (2015) 259 [arXiv:1506.03751] [INSPIRE].

[14] C. Grojean, E. Salvioni and R. Torre, A weakly constrained $W^{\prime}$ at the early LHC, JHEP 07 (2011) 002 [arXiv:1103.2761] [InSPIRE].

[15] L.A. Anchordoqui et al., Stringy origin of diboson and dijet excesses at the LHC, Phys. Lett. B 749 (2015) 484 [arXiv:1507.05299] [INSPIRE].

[16] C.-W. Chiang, H. Fukuda, K. Harigaya, M. Ibe and T.T. Yanagida, Diboson resonance as a portal to hidden strong dynamics, JHEP 11 (2015) 015 [arXiv:1507.02483] [INSPIRE].

[17] Z.-W. Wang, F.S. Sage, T.G. Steele and R.B. Mann, Can an asymptotically-safe conformal $\mathrm{U}(1)^{\prime}$ model address the LHC diboson excess?, arXiv:1511.02531 [INSPIRE].

[18] A. Sajjad, Understanding diboson anomalies, Phys. Rev. D 93 (2016) 055028 [arXiv: 1511.02244] [INSPIRE].

[19] B.A. Dobrescu and P.J. Fox, Signals of a $2 \mathrm{TeV} W^{\prime}$ boson and a heavier $Z^{\prime}$ boson, arXiv: 1511.02148 [INSPIRE]. 
[20] B.C. Allanach, P.S.B. Dev and K. Sakurai, ATLAS diboson excess could be an R-parity violating dismuon excess, Phys. Rev. D 93 (2016) 035010 [arXiv:1511.01483] [INSPIRE].

[21] J.H. Collins and W.H. Ng, A $2 \mathrm{TeV} W_{R}$, supersymmetry and the Higgs mass, JHEP 01 (2016) 159 [arXiv: 1510.08083] [INSPIRE].

[22] P. Ko and T. Nomura, $\mathrm{SU}(2)_{L} \times \mathrm{SU}(2)_{R}$ minimal dark matter with $2 \mathrm{TeV} W^{\prime}$, Phys. Lett. B 753 (2016) 612 [arXiv:1510.07872] [INSPIRE].

[23] A. Dobado, R.L. Delgado, F.J. Llanes-Estrada and D. Espriu, Comparing mesons and $W_{L} W_{L}$ TeV-resonances, in the proceedings of the Bled miniworkshop "Exploring hadron resonances", July 5-11, Bled, Slovenia (2015) [arXiv:1510.03761] [INSPIRE].

[24] D. Aristizabal Sierra, J. Herrero-Garcia, D. Restrepo and A. Vicente, Diboson anomaly: heavy Higgs resonance and QCD vectorlike exotics, Phys. Rev. D 93 (2016) 015012 [arXiv: 1510.03437] [INSPIRE].

[25] B.A. Arbuzov and I.V. Zaitsev, LHC diboson excesses as an evidence for a heavy $W W$ resonance, arXiv: 1510.02312 [INSPIRE].

[26] T. Li, J.A. Maxin, V.E. Mayes and D.V. Nanopoulos, Diboson excesses in leptophobic U(1) $)_{\mathrm{LP}}$ models from string theories, Phys. Rev. D 93 (2016) 045007 [arXiv:1509.06821] [INSPIRE].

[27] R.L. Awasthi, P.S.B. Dev and M. Mitra, Implications of the diboson excess for neutrinoless double Beta decay and lepton flavor violation in TeV scale left right symmetric model, Phys. Rev. D 93 (2016) 011701 [arXiv: 1509.05387] [INSPIRE].

[28] L. Bian, D. Liu, J. Shu and Y. Zhang, Interference effect on resonance studies and the diboson excess, arXiv: 1509.02787 [INSPIRE].

[29] C.-H. Chen and T. Nomura, Diboson excess in the Higgs singlet and vectorlike quark models, Phys. Rev. D 92 (2015) 115021 [arXiv: 1509. 02039] [INSPIRE].

[30] F.J. Llanes-Estrada, A. Dobado and R.L. Delgado, Describing $2 \mathrm{TeV}$ scale $W_{L} W_{L}$ resonances with unitarized effective theory, arXiv:1509.00441 [INSPIRE].

[31] S. Zheng, Discovery of scalar mixed with SM Higgs via diboson excess at the LHC, arXiv: 1508.06014 [INSPIRE].

[32] F.F. Deppisch et al., Reconciling the $2 \mathrm{TeV}$ excesses at the LHC in a linear seesaw left-right model, Phys. Rev. D 93 (2016) 013011 [arXiv: 1508.05940] [InSPIRE].

[33] C. Petersson and R. Torre, ATLAS diboson excess from low scale supersymmetry breaking, JHEP 01 (2016) 099 [arXiv: 1508.05632] [INSPIRE].

[34] S. Fichet, G. von Gersdorff and G. Gersdorff, Effective theory for neutral resonances and a statistical dissection of the ATLAS diboson excess, JHEP 12 (2015) 089 [arXiv:1508.04814] [INSPIRE].

[35] D. Goncalves, F. Krauss and M. Spannowsky, Augmenting the diboson excess for the LHC Run II, Phys. Rev. D 92 (2015) 053010 [arXiv:1508.04162] [InSPIRE].

[36] P. Coloma, B.A. Dobrescu and J. Lopez-Pavon, Right-handed neutrinos and the $2 \mathrm{TeV} W^{\prime}$ boson, Phys. Rev. D 92 (2015) 115023 [arXiv:1508.04129] [InSPIRE].

[37] P.S. Bhupal Dev and R.N. Mohapatra, Unified explanation of the eejj, diboson and dijet resonances at the LHC, Phys. Rev. Lett. 115 (2015) 181803 [arXiv:1508. 02277] [InSPIRE].

[38] P. Arnan, D. Espriu and F. Mescia, Interpreting a $2 \mathrm{TeV}$ resonance in $W W$ scattering, Phys. Rev. D 93 (2016) 015020 [arXiv:1508.00174] [InSPIRE]. 
[39] S.P. Liew and S. Shirai, Testing ATLAS diboson excess with dark matter searches at LHC, JHEP 11 (2015) 191 [arXiv: 1507.08273] [INSPIRE].

[40] H.M. Lee, D. Kim, K. Kong and S.C. Park, Diboson excesses demystified in effective field theory approach, JHEP 11 (2015) 150 [arXiv:1507.06312] [INSPIRE].

[41] L. Bian, D. Liu and J. Shu, Low scale composite Higgs model and $1.8 \sim 2$ TeV diboson excess, arXiv:1507.06018 [INSPIRE].

[42] W. Chao, ATLAS diboson excesses from the stealth doublet model, Phys. Lett. B 753 (2016) 117 [arXiv: 1507.05310] [INSPIRE].

[43] Y. Omura, K. Tobe and K. Tsumura, Survey of Higgs interpretations of the diboson excesses, Phys. Rev. D 92 (2015) 055015 [arXiv: 1507.05028] [INSPIRE].

[44] C.-H. Chen and T. Nomura, 2 TeV Higgs boson and diboson excess at the LHC, Phys. Lett. B 749 (2015) 464 [arXiv: 1507.04431] [InSPIRE].

[45] V. Sanz, On the compatibility of the diboson excess with a gg-initiated composite sector, arXiv: 1507.03553 [INSPIRE].

[46] G. Cacciapaglia, A. Deandrea and M. Hashimoto, Scalar hint from the diboson excess?, Phys. Rev. Lett. 115 (2015) 171802 [arXiv:1507.03098] [inSPIRE].

[47] B.A. Dobrescu and Z. Liu, Heavy higgs bosons and the $2 \mathrm{TeV} W^{\prime}$ boson, JHEP 10 (2015) 118 [arXiv: 1507.01923] [INSPIRE].

[48] A. Carmona, A. Delgado, M. Quirós and J. Santiago, Diboson resonant production in non-custodial composite Higgs models, JHEP 09 (2015) 186 [arXiv: 1507.01914] [INSPIRE].

[49] T. Abe, T. Kitahara and M.M. Nojiri, Prospects for spin-1 resonance search at $13 \mathrm{TeV}$ LHC and the ATLAS diboson excess, JHEP 02 (2016) 084 [arXiv:1507.01681] [INSPIRE].

[50] B.C. Allanach, B. Gripaios and D. Sutherland, Anatomy of the ATLAS diboson anomaly, Phys. Rev. D 92 (2015) 055003 [arXiv: 1507.01638] [INSPIRE].

[51] T. Abe, R. Nagai, S. Okawa and M. Tanabashi, Unitarity sum rules, three-site moose model and the ATLAS $2 \mathrm{TeV}$ diboson anomalies, Phys. Rev. D 92 (2015) 055016 [arXiv: 1507.01185] [INSPIRE].

[52] G. Cacciapaglia and M.T. Frandsen, Unitarity implications of a diboson resonance in the TeV region for Higgs physics, Phys. Rev. D 92 (2015) 055035 [arXiv: 1507.00900] [INSPIRE].

[53] Q.-H. Cao, B. Yan and D.-M. Zhang, Simple non-Abelian extensions of the standard model gauge group and the diboson excesses at the LHC, Phys. Rev. D 92 (2015) 095025 [arXiv: 1507.00268] [INSPIRE].

[54] J. Brehmer, J. Hewett, J. Kopp, T. Rizzo and J. Tattersall, Symmetry Restored in Dibosons at the LHC?, JHEP 10 (2015) 182 [arXiv: 1507.00013] [INSPIRE].

[55] A. Thamm, R. Torre and A. Wulzer, Composite heavy vector triplet in the ATLAS diboson excess, Phys. Rev. Lett. 115 (2015) 221802 [arXiv:1506.08688] [INSPIRE].

[56] Y. Gao, T. Ghosh, K. Sinha and J.-H. Yu, $\mathrm{SU}(2) \times \mathrm{SU}(2) \times \mathrm{U}(1)$ interpretations of the diboson and Wh excesses, Phys. Rev. D 92 (2015) 055030 [arXiv:1506.07511] [InSPIRE].

[57] J.A. Aguilar-Saavedra, Triboson interpretations of the ATLAS diboson excess, JHEP 10 (2015) 099 [arXiv:1506.06739] [INSPIRE]. 
[58] B.A. Dobrescu and Z. Liu, $W^{\prime}$ Boson near 2 TeV: predictions for Run 2 of the LHC, Phys. Rev. Lett. 115 (2015) 211802 [arXiv:1506.06736] [INSPIRE].

[59] K. Cheung, W.-Y. Keung, P.-Y. Tseng and T.-C. Yuan, Interpretations of the ATLAS diboson anomaly, Phys. Lett. B 751 (2015) 188 [arXiv:1506.06064] [INSPIRE].

[60] S.-S. Xue, Vectorlike $W^{ \pm}$_boson coupling at TeV and third family fermion masses, arXiv:1506.05994 [INSPIRE].

[61] D.B. Franzosi, M.T. Frandsen and F. Sannino, Diboson signals via Fermi scale spin-one states, Phys. Rev. D 92 (2015) 115005 [arXiv:1506.04392] [INSPIRE].

[62] J. Hisano, N. Nagata and Y. Omura, Interpretations of the ATLAS diboson resonances, Phys. Rev. D 92 (2015) 055001 [arXiv: 1506.03931] [INSPIRE].

[63] A. Alves, D.A. Camargo and A.G. Dias, Heavy Higgs coupled to vector-like quarks: strong CP problem, diboson excess and search prospects at the $14 \mathrm{TeV} L H C$, arXiv:1511.04449 [INSPIRE].

[64] L.J. Dixon, Calculating scattering amplitudes efficiently, hep-ph/9601359 [INSPIRE].

[65] D. Spehler and S.F. Novaes, Helicity wave functions for massless and massive spin-2 particles, Phys. Rev. D 44 (1991) 3990 [INSPIRE].

[66] S.F. Novaes and D. Spehler, Weyl-Van Der Waerden spinor technic for spin 3/2 fermions, Nucl. Phys. B 371 (1992) 618 [inSPIRE].

[67] L.D. Landau, On the angular momentum of a two-photon system, Dokl. Akad. Nauk Ser. Fiz. 60 (1948) 207 [INSPIRE].

[68] C.-N. Yang, Selection rules for the dematerialization of a particle into two photons, Phys. Rev. 77 (1950) 242 [INSPIRE].

[69] W.-Y. Keung, I. Low and J. Shu, Landau-Yang theorem and decays of a $Z^{\prime}$ boson into two $Z$ bosons, Phys. Rev. Lett. 101 (2008) 091802 [arXiv:0806.2864] [INSPIRE].

[70] S.N. Gninenko, A. Yu. Ignatiev and V.A. Matveev, Two photon decay of $Z^{\prime}$ as a probe of bose symmetry violation at the CERN LHC, Int. J. Mod. Phys. A 26 (2011) 4367 [arXiv: 1102.5702] [INSPIRE].

[71] M. Cacciari, L. Del Debbio, J.R. Espinosa, A.D. Polosa and M. Testa, A note on the fate of the Landau-Yang theorem in non-Abelian gauge theories, Phys. Lett. B 753 (2016) 476 [arXiv: 1509.07853] [INSPIRE].

[72] K.S. Babu, C.F. Kolda and J. March-Russell, Leptophobic U(1)s and the R(b)-R(c) crisis, Phys. Rev. D 54 (1996) 4635 [hep-ph/9603212] [INSPIRE].

[73] A. Crivellin, G. D'Ambrosio and J. Heeck, Addressing the LHC flavor anomalies with horizontal gauge symmetries, Phys. Rev. D 91 (2015) 075006 [arXiv:1503.03477] [InSPIRE].

[74] P. Fileviez Perez and M.B. Wise, Baryon and lepton number as local gauge symmetries, Phys. Rev. D 82 (2010) 011901 [Erratum ibid. D 82 (2010) 079901] [arXiv: 1002.1754] [INSPIRE].

[75] T.R. Dulaney, P. Fileviez Perez and M.B. Wise, Dark matter, baryon asymmetry and spontaneous B and L breaking, Phys. Rev. D 83 (2011) 023520 [arXiv:1005.0617] [INSPIRE].

[76] P. Fileviez Perez and M.B. Wise, Breaking local baryon and lepton number at the TeV scale, JHEP 08 (2011) 068 [arXiv:1106.0343] [inSPIRE]. 
[77] M. Duerr, P. Fileviez Perez and M.B. Wise, Gauge theory for baryon and lepton numbers with leptoquarks, Phys. Rev. Lett. 110 (2013) 231801 [arXiv:1304.0576] [INSPIRE].

[78] E. Accomando, A. Belyaev, L. Fedeli, S.F. King and C. Shepherd-Themistocleous, $Z^{\prime}$ physics with early LHC data, Phys. Rev. D 83 (2011) 075012 [arXiv:1010.6058] [InSPIRE].

[79] Q.-H. Cao, Z. Li, J.-H. Yu and C.P. Yuan, Discovery and identification of $W^{\prime}$ and $Z^{\prime}$ in $\mathrm{SU}(2) \times \mathrm{SU}(2) \times \mathrm{U}(1)$ models at the LHC, Phys. Rev. D 86 (2012) 095010 [arXiv: 1205.3769] [INSPIRE].

[80] CMS collaboration, Search for resonant t $\bar{t}$ production in proton-proton collisions at $\sqrt{s}=8$ TeV, Phys. Rev. D 93 (2016) 012001 [arXiv:1506.03062] [InSPIRE].

[81] ATLAS collaboration, $A$ search for $t \bar{t}$ resonances using lepton plus jets events in proton-proton collisions at $\sqrt{s}=8 \mathrm{TeV}$ with the ATLAS detector, ATLAS-CONF-2015-009 (2015).

[82] CMS collaboration, Search for resonances and quantum black holes using dijet mass spectra in proton-proton collisions at $\sqrt{s}=8 \mathrm{TeV}$, Phys. Rev. D 91 (2015) 052009 [arXiv: 1501.04198] [INSPIRE].

[83] ATLAS collaboration, Search for new phenomena in the dijet mass distribution using pp collision data at $\sqrt{s}=8 \mathrm{TeV}$ with the ATLAS detector, Phys. Rev. D 91 (2015) 052007 [arXiv: 1407.1376] [INSPIRE].

[84] ATLAS collaboration, Search for resonances with boson-tagged jets in $3.2 \mathrm{fb}^{-1}$ of pp collisions at $\sqrt{s}=13 \mathrm{TeV}$ collected with the ATLAS detector, ATLAS-CONF-2015-073 (2015).

[85] CMS collaboration, Search for massive resonances decaying into pairs of boosted $W$ and $Z$ bosons at $\sqrt{s}=13 \mathrm{TeV}$, CMS-PAS-EXO-15-002 (2015).

[86] M. Peskin and T. Takeuchi, Estimation of oblique electroweak corrections, Phys. Rev. D 46 (1992) 381 [INSPIRE].

[87] http://project-gfitter.web.cern.ch/project-gfitter/publications.html. 